\title{
Influence of comorbidities and clinical prediction model on neurological prognostication post out-of- hospital cardiac arrest
}

\author{
Weiting Huang, ${ }^{1}$ Gary Kuan Wee Teo, ${ }^{1}$ Jack Wei-Chieh Tan, ${ }^{1}$ Nur Shahidah Ahmad, ${ }^{2}$ \\ Hwee Hong Koh, ${ }^{1}$ Marcus Eng Hock Ong ${ }^{2}$
}

${ }^{1}$ Cardiology, National Heart Centre Singapore, Singapore, Singapore

${ }^{2}$ Emergency Medicine, Singapore General Hospital, Singapore, Singapore

Correspondence to DrWeiting Huang; huang. weiting@singhealth.com.sg

Received 7 February 2018 Revised 16 May 2018 Accepted 25 May 2018
Check for updates

To cite: Huang W, Teo GKW, Tan JW-C, et al. Heart Asia 2018;10:e011016. doi:10.1136/

heartasia-2018-011016

\section{ABSTRACT}

Background Survival with good neurological function post out-of-hospital cardiac arrest (OHCA), defined as cerebral performance category (CPC) $1-2$, ranges from $1.6 \%$ to $3 \%$ in Asia. We aim to study the influence of comorbidities and peri-OHCA event factors on neurological recovery and develop a model that can help clinicians predict neurological function among patients with post-OHCA admitted to the hospital.

Methods This was a retrospective cohort study. All patients admitted post-OHCA from 1 January 2011 to 31 December 2015 to a tertiary centre were identified through the hospital OHCA registry. Patients who survived till hospital admission were included. Logistic regression was used to identify patient and peri-arrest factors that were significantly associated with survival with CPC 1-2. The significant factors for survival with CPC 1-2 were then put into a multivariable model and the discriminative ability was tested using the receiver operator characteristic (ROC) curve. Calibration and internal validation of the model were also performed. External validation in a small prospective cohort was also performed.

Results In our derivation cohort of 129 patients, $30.23 \%$ survived with CPC $1-2$. Significant factors associated with survival with good neurological outcomes were age-adjusted Charlson Comorbidity Index $\leq 5$, time to first return of spontaneous circulation $\leq 40 \mathrm{~min}$, the presence of immediate bystander cardiopulmonary resuscitation and shockable rhythms. We also developed a nomogram which showed good internal (ROC curve $0.84 ; 95 \% \mathrm{Cl} 0.77$ to 0.91 ) and external validation (ROC curve $0.90 ; 95 \% \mathrm{Cl} 0.81$ to 1.00).

\section{INTRODUCTION}

Out-of-hospital cardiac arrest (OHCA) is an acute and often fatal condition that constitutes a major challenge to global healthcare. In Asia, the overall survival to hospital discharge varied from $0.5 \%$ to $8.5 \%$, and survival with good neurological function ranged from $1.6 \%$ to $3 \% .{ }^{1}$ In patients who initially achieve return of spontaneous circulation (ROSC) after OHCA, the significant morbidity attributable to the cerebral and cardiac dysfunction that accompanies prolonged whole-body ischaemia, may be irreversible. $^{2}$ Targeted temperature management (TTM) with mild hypothermia of $32^{\circ} \mathrm{C}-36^{\circ} \mathrm{C}^{3}$ is an important tool for the treatment of postanoxic coma after cardiopulmonary resuscitation (CPR). It has been shown to reduce mortality and has improved neurological outcomes after cardiac arrest and should be offered to all patients with eligible OHCA.

The presence of chronic conditions, such as heart disease, hypertension and diabetes, is known to increase with age, and evidence suggests that the majority of patients with OHCA have at least one comorbid condition. ${ }^{45}$ However, to date the influence of pre-arrest comorbidities on outcomes following OHCA has not been well established. Some studies have demonstrated a deleterious association between chronic health conditions and OHCA outcomes, ${ }^{4}{ }^{6}$ while others have reported that comorbid factors do not influence outcomes. ${ }^{78}$ Comorbid diseases are illnesses that are typically chronic in nature and have a significant impact on both short-term and long-term mortality. ${ }^{69}$ These are important confounders and may affect interpretation of cardiac arrest studies. ${ }^{10}$

The likelihood of survival with good neurological function is an important consideration for family members of victims of postcardiac arrest. There are currently few prediction models available to prognosticate patients' postcardiac arrest ${ }^{11-13}$; some were initially developed for inpatient cardiac arrest while others require biochemistry results which may be cumbersome to use.

In this study, we aimed to study the impact of comorbidities measured by the age-adjusted Charlson Comorbidity Index (ACCI) on survival with good neurological function, defined as cerebral performance category (CPC) 1-2 in patients with OHCA and also develop a prediction tool, incorporating significant peri-arrest factors that can be used to help inform physicians and family members on probabilities of survival with good neurological outcome when patients survive to hospital admission post-OHCA.

\section{METHOD}

\section{Study design and settings}

This retrospective cohort was carried out at the Singapore General Hospital from 2011 to 2015. Singapore is a multiracial Southeast Asian country which has a population of approximately 5.5 million people. The Singapore General Hospital has a total of 1597 inpatient beds, which hosts 185 cardiology-dedicated beds including 25 cardiothoracic and cardiology intensive care unit beds, with a 24 hours angiography suite for ST elevation myocardial infarction and other cardiology emergencies. There was also a prospective observational arm used as the 
external validation cohort. This study was conducted in compliance with the Declaration of Helsinki.

\section{Participants}

All cases of OHCA conveyed to Singapore General Hospital during the period of 1 January 2011 to 31 December 2015 were identified through the hospital's cardiac arrest registry. The Singapore General Hospital participates in the Pan-Asian Resuscitation Outcomes Study (PAROS) ${ }^{14}$ and their definition of OHCA was used, that is, out-of-hospital event in a patient who unresponsive, apnoeic and pulseless. We included all patients above the age of 18, who remained comatose (ie, lack of meaningful response to verbal commands) upon ROSC and survived to hospital admission. Patients who did not survive to hospital admission, transferred to another hospital and for whom resuscitation was not attempted were excluded. We also collected data from a prospective cohort admitted with cardiac arrest between January 2016 and October 2017 for external validation of the prediction tool.

\section{Data collection and categorisation}

Patients were identified through the hospital's cardiac arrest registry. Data on the following variables were collected by an investigator who was blinded to patient outcomes: baseline demographics, comorbid conditions and computation of ACCI, the presence of immediate bystander CPR, use of mechanical $\mathrm{CPR}$, first documented heart rhythm (ventricular tachycardia (VT)/ventricular fibrillation (VF) or pulseless electrical activity/ asystole), duration from time of collapse to first ROSC, decision for immediate coronary angiography and cause of collapse. The ACCI is the most widely used comorbidity index. ${ }^{15} 16$ It was developed to predict the 1-year mortality among 604 patients based on comorbidity data obtained from a hospital chart review in a single US hospital. ${ }^{15}$ It contains 19 issues including diabetes with diabetic complications, congestive heart failure, peripheral vascular disease, chronic pulmonary disease, mild and severe liver disease, hemiplegia, renal disease, leukaemia, lymphoma, metastatic tumour and AIDS, each of which was weighted according to their potential influence on mortality. It was further adjusted for age by adding one extra point for each decade of life after 50 years of a. ${ }^{17} \mathrm{~A}$ second independent investigator blinded to the above clinical data collected information on the outcome, that is, CPC score categorised as good (1-2) and poor (3-5) at discharge from the hospital and TTM therapy.

We also prospectively collected all patients who were admitted to Singapore General Hospital and survived to hospital admission from January 2016 to October 2017 to test and validate our prediction tool.

\section{Data analysis}

Clinical and treatment characteristics of patients with and without survival with CPC 1-2 were compared using independent sample t-tests (continuous variables) or $\mathrm{X}^{2}$ tests (categorical or dichotomous variables). We chose a significance level of 0.05 to determine factors to be put in the multivariable model. Multivariate logistic regression analyses were then applied to relate baseline demographics and factors peri-arrest to the outcome of survival with CPC 1-2. Parameters with $\mathrm{p}$ values of $<0.05$ included the multivariate analysis are as follows: $\mathrm{ACCI} \leq 5$, the presence of immediate bystander CPR, shockable rhythms due to VT or VF and TTM. These predictors corresponded with the previous literature, except for the new factor for which we are exploring, which is the presence or absence of multiple comorbidities, ACCI $\leq 5$.
To create the prediction model, we looked at pre-arrest and peri-arrest factors present in our study that were significantly associated with survival with CPC 1-2. TTM was not included in the base model as it was a post-cardiac arrest therapy and should be applied to all patients eligible.

We input factors of ACCI $\leq 5$, time from collapse to first ROSC $\leq 40 \mathrm{~min}$, the presence of immediate bystander CPR and shockable rhythms due to VT or VF into the model. Discriminative ability (ie, the ability of the model to distinguish patients who will survive with CPC 1-2 from patients who may not) of the model was quantified by the area under the receiver operating characteristic (ROC) curve (ROC area). Calibration of the final model was determined by comparing the predicted risks with the observed proportions of patients with survival with CPC1-2, among five risk groups (and performing the Hosmer-Lemeshow test). The regression coefficients (b-coefficients) were divided by the smallest coefficient in the model. ${ }^{18}$

The final model with the adjusted coefficients, with rounding, was presented as a nomogram to facilitate clinical application. Finally, the sum scores based on the absence or presence of the clinical factors were related to predicted risks. For internal validation of the model, we performed bootstrapping analysis.

As TTM is an established factor known to improve survival with good neurological prognosis, we performed a sensitivity analysis to determine if addition of the factor of TTM to our base model consisting of pre-arrest and peri-arrest factors will significantly improve the performance of the model in terms of its ROC curve.

We subsequently validated our model in a prospective cohort of patients with OHCA who survived to hospital admission between January 2016 and October 2017. The performance of the model was further tested with K-fold cross-validation combining both derivation and validation cohorts.

A commercial statistical package (Stata V.14) was used for statistical analysis of the data. Statistical significance was set at $\mathrm{p}<0.05$.

\section{RESULTS}

Of 129 patients, 60 patients (46.5\%) patients underwent TTM and 39 patients (30.23\%) survived with good CPC score of 1-2.

Univariate as well as multivariate analysis of the factors associated with survival with good neurological function is shown in tables 1 and 2. The discriminative value (ROC area) of the model, consisting factors of ACCI $\leq 5$, time from collapse to first ROSC $\leq 40 \mathrm{~min}$, the presence of immediate bystander CPR and shockable rhythms due to VT or VF collapse, was 0.84 (95\% CI 0.77 to 0.91$)$. Bootstrapping analysis for internal validation and to avoid optimism showed an area under the curve of 0.81 (95\% CI 0.73 to 0.89 ). This good calibration of the derivation cohort is also confirmed by a non-significant Hosmer-Lemeshow test $(\mathrm{p}=0.87)$.

For external validation, we prospectively collected data of 31 consecutive patients with OHCA who survived to hospital admission from January 2016 to October 2017. The observed frequencies of survival with good neurological outcome of CPC 1-2 corroborated well with the prediction model when the model score is 4 and above. For lower scores, the model overestimates the probability of survival with good neurological outcomes, but that may be due to the smaller sample size of the validation cohort. The area of the ROC curve using the nomogram for the validation cohort was 0.90 (95\%CI 0.81 to 1.00$)$.

We also used K-fold cross-validation of the entire cohort, combining the derivation cohort and validation cohort to assess 
Table 1 Baseline demographics of study population and patients who survived with cerebral performance category (CPC) 1-2

\begin{tabular}{|c|c|c|c|}
\hline & All patients $(n=129)$ & Survival with CPC 1-2 $(n=39)$ & $P$ values \\
\hline \multicolumn{4}{|l|}{ Age group } \\
\hline 19-55 years & $38(29.46 \%)$ & $18(46.15 \%)$ & Ref \\
\hline$>55-65$ years & $38(29.46 \%)$ & $13(33.33 \%)$ & 0.245 \\
\hline$>65-75$ years & $34(26.36 \%)$ & $6(15.38 \%)$ & 0.010 \\
\hline$>75$ years & $19(14.73 \%)$ & $2(5.13 \%)$ & 0.013 \\
\hline Female & $28(21.71 \%)$ & $9(23.08 \%)$ & 0.804 \\
\hline \multicolumn{4}{|l|}{ Race } \\
\hline Chinese & $92(71.32 \%)$ & $27(69.23 \%)$ & Ref \\
\hline Malay & $10(7.75 \%)$ & $3(7.69 \%)$ & 0.966 \\
\hline Indian & $10(7.75 \%)$ & - & \\
\hline Others & $17(13.18 \%)$ & $9(23.08 \%)$ & 0.064 \\
\hline \multicolumn{4}{|l|}{ Body mass index } \\
\hline$<18.5$ & $18(13.95 \%)$ & $9(23.08 \%)$ & 0.487 \\
\hline $18.5-22.9$ & $23(17.83 \%)$ & $9(23.08 \%)$ & Ref \\
\hline $23-26.9$ & $48(37.21 \%)$ & $12(30.77 \%)$ & 0.226 \\
\hline$\geq 27$ & $40(31.01 \%)$ & $9(23.08 \%)$ & 0.164 \\
\hline Hypertension & $79(61.24 \%)$ & $20(51.28 \%)$ & 0.129 \\
\hline Diabetes mellitus & $44(34.11 \%)$ & $9(23.08 \%)$ & 0.085 \\
\hline History of stroke & $50(38.76 \%)$ & $14(35.90 \%)$ & 0.661 \\
\hline$\geq$ Stage 3 chronic kidney disease & $65(50.39 \%)$ & $15(38.46 \%)$ & 0.077 \\
\hline End-stage renal failure requiring dialysis & $7(5.43 \%)$ & $3(7.69 \%)$ & 0.460 \\
\hline Known history of ischaemic heart disease & $52(40.31 \%)$ & $11(28.21 \%)$ & 0.068 \\
\hline Dementia & 1 & 0 & - \\
\hline Connective tissue disease & 1 & 0 & - \\
\hline Peptic ulcer disease & $11(8.53 \%)$ & $2(5.13 \%)$ & 0.372 \\
\hline Chronic respiratory disease & $28(21.71 \%)$ & $9(23.08 \%)$ & 0.804 \\
\hline Liver disease & $31(24.03 \%)$ & $7(17.95 \%)$ & 0.290 \\
\hline Cancer & $3(2.33 \%)$ & 0 & NA \\
\hline Age-adjusted Charlson Ccomorbidity Index (CCI) & $4.10 \pm 2.39 .68 \%)$ & $2.61 \pm 1.85$ & \\
\hline $\mathrm{CCl} 0-2$ & $35(27.13 \%)$ & $21(53.85 \%)$ & Ref \\
\hline $\mathrm{CCl} 3-5$ & $57(44.19 \%)$ & $15(38.46 \%)$ & 0.059 \\
\hline $\mathrm{CCl}>5$ & $37(28.68 \%)$ & $3(7.69 \%)$ & $<0.001$ \\
\hline Presence of bystander cardiopulmonary resuscitation & $64(49.61 \%)$ & $26(66.67 \%)$ & 0.0012 \\
\hline Time to return of spontaneous circulation (min) & $38.14 \pm 22.87$ & $24.15 \pm 16.66$ & $<0.001$ \\
\hline \multicolumn{4}{|l|}{ Time to first return of spontaneous circulation (min) } \\
\hline $0-10$ & $12(9.30 \%)$ & $7(17.95 \%)$ & Ref \\
\hline$>10-20$ & $21(16.28 \%)$ & $15(38.46 \%)$ & 0.445 \\
\hline$>20-30$ & $26(20.16 \%)$ & $8(20.51 \%)$ & 0.113 \\
\hline$>30-40$ & $20(15.50 \%)$ & $6(15.38 \%)$ & 0.120 \\
\hline$>40-50$ & $20(15.50 \%)$ & $1(2.56 \%)$ & 0.005 \\
\hline$>50-60$ & $14(10.85 \%)$ & 0 & - \\
\hline$>60$ & $16(12.40 \%)$ & $2(5.13 \%)$ & 0.017 \\
\hline Ventricular tachycardia/ventricular fibrillation collapse & $74(57.36 \%)$ & $31(79.49 \%)$ & 0.001 \\
\hline Collapse due to myocardial infarction & $90(69.77 \%)$ & $27(69.23 \%)$ & 0.930 \\
\hline Went for direct coronary catheterisation & $84(66.14 \%)$ & $29(74.36 \%)$ & 0.195 \\
\hline Targeted temperature management & $69(53.49 \%)$ & $28(71.79 \%)$ & 0.007 \\
\hline
\end{tabular}

the performance of the model. The sensitivity and specificity of the model are $66.00 \%$ and $86.36 \%$, respectively, and the positive and negative predictive values are $68.75 \%$ and $84.82 \%$, respectively.

The tables 3 and 4 show that calibration (ie, agreement between predicted risks and observed frequencies of survival with CPC 1-2) of the model was good and also observed frequencies in the validation cohort.

As TTM is a known therapy to improve neurological outcomes, a sensitivity analysis was performed to determine if its inclusion will affect the model. We compared the ROC curve of our base model including the four factors (ACCI $\leq 5$, time to ROSC $\leq 40$ min, the presence of immediate bystander CPR and a shockable rhythm) to the same base model with the additional factor of receiving TTM (five factors). The ROC area of the base model is 0.843 while that of the base model plus the additional factor of TTM was 0.856 , with a non-significant $\mathrm{p}$ value of 0.341 . Hence, the discriminative ability of both models was equal. In view of parsimony, we did not include TTM as a variable on our final nomogram (see figure 1 ). 


\section{Original research}

Table 2 Univariate and multivariate analysis of factors associated with survival with cerebral performance category (CPC) 1-d 2

\begin{tabular}{|c|c|c|c|c|}
\hline & \multicolumn{2}{|c|}{ Univariate logistic regression } & \multicolumn{2}{|c|}{ Multivariate logistic regression } \\
\hline & OR $(95 \% \mathrm{Cl})$ & $P$ values & OR $(95 \% \mathrm{Cl})$ & $P$ values \\
\hline Age $<65$ years & 3.87 (1.61 to 9.34$)$ & 0.003 & - & - \\
\hline Female & 1.12 (0.45 to 2.76$)$ & 0.804 & - & - \\
\hline \multicolumn{5}{|l|}{ Race } \\
\hline Chinese & 1.0 & Ref & - & - \\
\hline Malay & 1.03 (0.25 to 4.29$)$ & 0.966 & & \\
\hline Indian & 1.0 & NS & & \\
\hline Others & 2.71 (0.94 to 7.76$)$ & 0.064 & & \\
\hline \multicolumn{5}{|l|}{ Body mass index } \\
\hline$<18.5$ & 1.94 (0.59 to 6.35$)$ & 0.271 & - & - \\
\hline $18.5-25$ & 1.0 & & & \\
\hline$>25$ & 0.66 (0.25 to 1.73$)$ & 0.399 & & \\
\hline Hypertension & 0.55 (0.26 to 1.19$)$ & 0.129 & - & - \\
\hline Diabetes mellitus & 0.47 (0.20 to 1.11$)$ & 0.085 & - & - \\
\hline History of stroke & 0.84 (0.38 to 1.83$)$ & 0.661 & - & - \\
\hline$\geq$ Stage 3 chronic kidney disease & 0.50 (0.23 to 1.08$)$ & 0.077 & - & - \\
\hline End-stage renal failure requiring dialysis & 1.79 (0.38 to 8.41$)$ & 0.460 & - & - \\
\hline Known history of ischaemic heart disease & $0.47(0.21$ to 1.06$)$ & 0.068 & - & - \\
\hline Age-adjusted Charlson Comorbidity Index $\leq 5$ & 7.28 (2.08 to 25.49$)$ & 0.002 & 5.14 (1.33 to 19.89$)$ & 0.018 \\
\hline Presence of bystander cardiopulmonary resuscitation & $2.74(1.25$ to 6.01$)$ & 0.012 & $2.84(1.09$ to 7.40$)$ & 0.033 \\
\hline Time to first return of spontaneous circulation $\leq 40 \mathrm{~min}$ & 7.01 (2.95 to 16.67$)$ & $<0.001$ & $6.92(4.93$ to 16.47$)$ & $<0.001$ \\
\hline Ventricular tachycardia/ventricular fibrillation collapse & 4.23 (1.76 to 10.21$)$ & 0.001 & 2.59 (1.04 to 7.19$)$ & 0.048 \\
\hline Collapse due to myocardial Infarction & 0.96 (0.43 to 2.18$)$ & 0.930 & - & - \\
\hline Went for direct coronary catheterisation & 1.74 (0.75 to 4.02$)$ & 0.195 & - & - \\
\hline Underwent targeted temperature management & 3.04 (1.35 to 6.85$)$ & 0.007 & 2.60 (0.97 to 6.96$)$ & 0.057 \\
\hline
\end{tabular}

The nomogram to aid simple clinical application is shown in table 3.

\section{DISCUSSION}

In our study, $30.23 \%$ of all patients admitted for OHCA survived with good neurological outcomes. ACCI $\leq 5$, time to ROSC $\leq 40 \mathrm{~min}$, the presence of immediate bystander CPR, a shockable rhythm and TTM were significantly associated with survival with good neurological outcomes.

Patients who had TTM had an OR 3.04 (95\% CI 1.35 to 6.85 ) of surviving with a good neurological outcome compared with those who did not receive the treatment. Despite this, only $46.5 \%$ of our patient received TTM therapy. This may be due to concerns regarding underlying comorbidities and complications such as electrolyte and fluid shifts associated with TTM. ${ }^{19}$ Previous studies have also showed that there is heterogeneity in the use of TTM therapy in patients with OHCAs, with uptake ranging from $20 \%$ to $40 \% .^{20}{ }^{21}$ Factors cited include logistical or resource issues, the perceived lack of evidence or consensus within individual ICU teams or not considering it at all. We hope to improve the uptake of this therapy in our programme through continual education and resource management.

Table 3 Scoring system of prediction model

\begin{tabular}{ll}
\hline Factor & Value \\
\hline Age-adjusted Charlson Comorbidity Index $\leq 5$ & +2 \\
Time to first return of spontaneous circulation $\leq 40 \mathrm{~min}$ & +2 \\
Immediate bystander cardiopulmonary resuscitation & +1 \\
Shockable rhythm & +1 \\
Maximum score attainable & 6 \\
\hline
\end{tabular}

The most important question post-OHCA for physician and family is the likelihood of survival with good neurological recovery. Currently available scores such as the OHCA score ${ }^{22}$ and Cardiac Arrest Hospital Prognosis score ${ }^{11}$ are useful but contain complex mathematical formulas and require biochemistry results which have a turn-around time; this may make the tool less widely in acute situations. On the other hand, simpler calculators like the Good Outcome Following Attempted Resuscitation (GO-FAR) score have been validated for in-hospital but not OHCA. Similar to the GO-FAR score, ${ }^{23}$ our tool consists mainly of premorbid clinical characteristics and peri-resuscitation information.

We chose the age-adjusted comorbidity index as an overall measure of comorbidity and frailty as it takes into account the

Table 4 Observed and predicted probabilities of survival with cerebral performance category (CPC) 1-2 based on prediction score

\begin{tabular}{|c|c|c|c|c|}
\hline \multirow[b]{2}{*}{$\begin{array}{l}\text { Probability of } \\
\text { survival with } \\
\text { CPC 1-2 }\end{array}$} & \multirow[b]{2}{*}{$\begin{array}{l}\text { Model } \\
\text { scores }\end{array}$} & \multicolumn{2}{|c|}{ Derivation cohort $(n=129)$} & \multirow[t]{2}{*}{$\begin{array}{l}\text { Validation } \\
\text { cohort }(n=31)\end{array}$} \\
\hline & & $\mathrm{n} / \mathrm{N}(\%)$ & $\begin{array}{l}\text { Predicted } \% \text { survival } \\
\text { with CPC } 1-2 \text { based } \\
\text { on model calculation } \\
(95 \% \mathrm{Cl})\end{array}$ & \\
\hline$<5 \%$ & $0-1$ & $0 / 19(0)$ & 2.95 (1.16 to 3.23$)$ & $0 / 4$ \\
\hline $5 \%-25 \%$ & $2-3$ & $6 / 48(12.50 \%)$ & 11.00 (5.71 to 19.30$)$ & $0 / 8$ \\
\hline$>25 \%-45 \%$ & 4 & $9 / 25(35.00 \%)$ & 30.78 (30.78 to 32.21$)$ & $3 / 8(37.50 \%)$ \\
\hline$>45 \%-65 \%$ & 5 & $7 / 16(43.75 \%)$ & 55.15 (55.15 to 57.41$)$ & $2 / 4(50.00 \%)$ \\
\hline$>65 \%$ & 6 & $17 / 21(80.95 \%)$ & 77.72 & $6 / 7(85.71 \%)$ \\
\hline
\end{tabular}




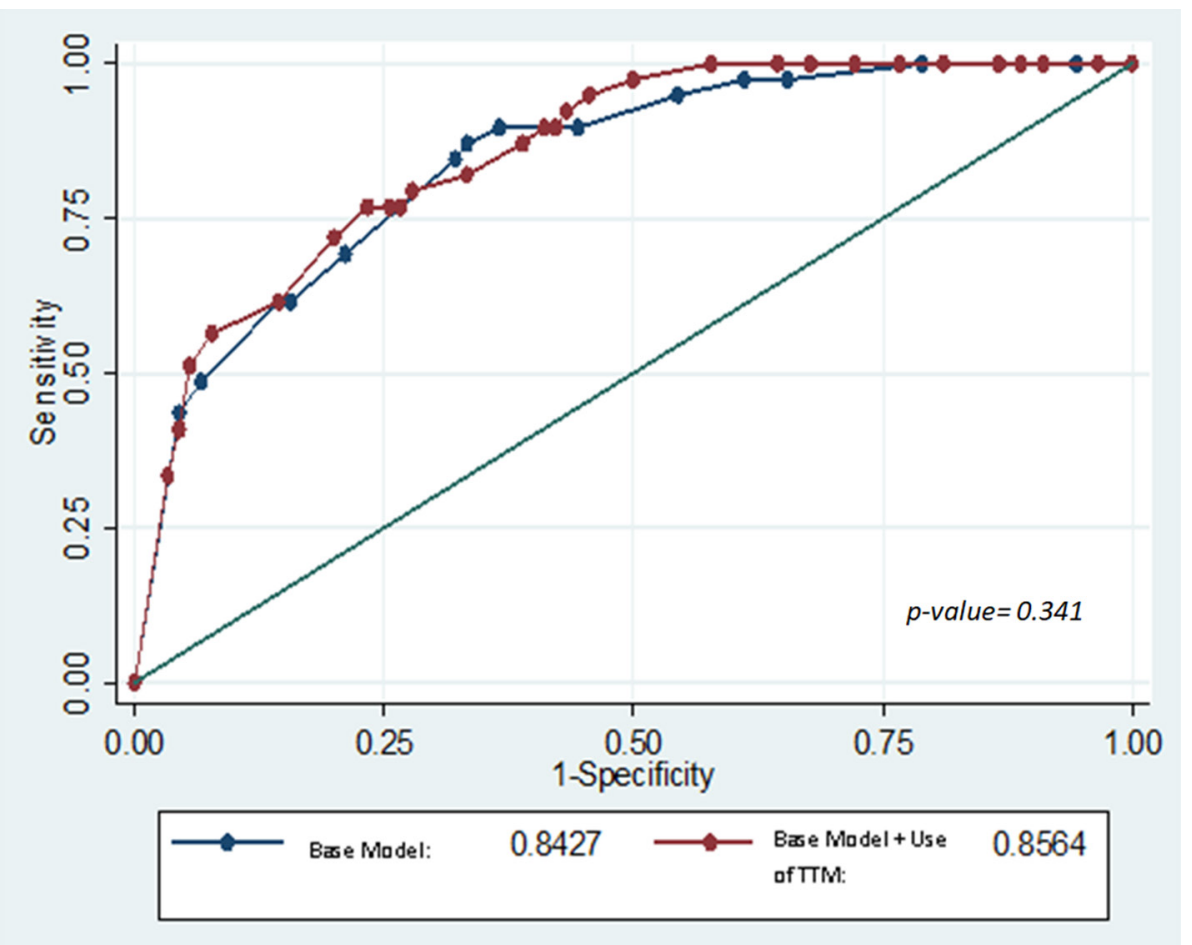

Figure 1 Comparison of receiver operating curves of base model (age-adjusted Charlson Comorbidity Index $\leq 5$, time to return of spontaneous circulation $\leq 40 \mathrm{~min}$, the presence of immediate bystander cardiopulmonary resuscitation and a shockable rhythm) and base model+targeted temperature management.

interaction of age and comorbidities. This is especially important as patients with OHCAs come from heterogeneous age groups, and our study suggests that it may not be a single comorbid, but rather the constellation of comorbidities, that reflects the patient's physiological reserve to respond to cardiac arrest. As age is included in the sum aggregate of the ACCI, we did not include age in our model due to potential confounding.

Based on our nomogram, a patient with all the good prognostic factors of ACCI $\leq 5$, time to ROSC $\leq 40 \mathrm{~min}$, the presence of immediate bystander CPR and a shockable rhythm will have a probability of survival with good neurological outcome of $>65 \%$, while one without any of the above will have a probability of $<5 \%$. We also tested our model in a small prospective cohort, demonstrating external validation of the tool.

Strengths of our study are that these patients are obtained from a cardiac arrest registry and had complete follow-up and data collection on their risk factors. We also blinded the investigators performing data collection, so as to reduce observer bias.

Our limitations include a small sample size; however, the number of events (survival with CPC 1-2) was sufficient to explore the four factors and to generate a nomogram based on the 1 factor to 10 events rule. This was also a single-centre study and there may be the presence of unmeasurable confounders which have not been tested in our analysis. To overcome this, we intend collaborate with the ongoing prospective PAROS registry and seek to validate our prediction model on separate larger cohorts to determine external validity.

\section{CONCLUSION}

Comorbidities have a significant influence on survival with good neurological function post-OHCA and should be taken into consideration during care of such patients. We have proposed a tool, which takes into account the influence of comorbidities, to help inform physicians and family on probability of survival with good neurological recovery. More research is needed to validate this tool in separate larger cohorts of patients with OHCA.

\section{Key messages}

What is already known about this subject?

- Rates of survival with good neurological outcomes post outof-hospital cardiac arrest remain low over the years. Targeted temperature management helps to improve neurological outcomes.

What does this study add?

- Age-adjusted Charlson Comorbidity index is a strong predictor of survival with good neurological outcome post out-of-hospital cardiac arrest, and it may be the combined burden of multiple comorbidities, rather than individual weighted ones, that affect neurological prognosis

- We developed a probability tool that helps clinicians calculate the likelihood of survival with good neurological outcome post out-of-hospital cardiac arrest based on age-adjusted Charlson comorbidity index and peri-arrest factors.

- Targeted temperature management improved neurological outcomes in our cohort, but did not improve receiver operating curve when compared to a base probability model, in determining good neurological outcomes.

How might this impact on clinical practice?

- This score aids neurological prognostication and discussion for further invasive procedures in patients who survive outof-hospital cardiac arrest. 


\section{Original research}

Contributors All authors have participated in the work and have reviewed and agree with the content of the article.

Funding The authors have not declared a specific grant for this research from any funding agency in the public, commercial or not-for-profit sectors.

Disclaimer None of the article contents are under consideration for publication in any other journal or have been published in any journal. No portion of the text has been copied from other material in the literature (unless in quotation marks, with citation). The authors are aware that it is their responsibility to obtain permission for any figures or tables reproduced from any prior publications and to cover fully any costs involved. Such permission must be obtained prior to final acceptance.

Competing interests None declared.

Patient consent Not required.

Ethics approval Singhealth Centralised Institutional Review Board.

Provenance and peer review Not commissioned; externally peer reviewed.

Data sharing statement Statistical code and raw data set can be made available upon request via contacting the corresponding author.

(c) Article author(s) (or their employer(s) unless otherwise stated in the text of the article) 2018. All rights reserved. No commercial use is permitted unless otherwise expressly granted.

\section{REFERENCES}

1. Ong ME, Shin SD, De Souza NN, et al. Outcomes for out-of-hospital cardiac arrests across 7 countries in Asia: the Pan Asian Resuscitation Outcomes Study (PAROS). Resuscitation 2015:96:100-8.

2. Neumar RW, Nolan JP, Adrie C, et al. Post-cardiac arrest syndrome. Circulation 2008; 118:2452-83.

3. Morrison LJ, Deakin CD, Morley PT, et al. Part 8: Advanced life support: 2010 International Consensus on Cardiopulmonary Resuscitation and Emergency Cardiovascular Care Science With Treatment Recommendations. Circulation 2010;122(16 Suppl 2):S345-S421.

4. Carew HT, Zhang W, Rea TD. Chronic health conditions and survival after out-ofhospital ventricular fibrillation cardiac arrest. Heart 2007;93:728-31.

5. Søholm H, Hassager C, Lippert F, et al. Factors associated with successful resuscitation after out-of-hospital cardiac arrest and temporal trends in survival and comorbidity. Ann Emerg Med 2015;65:523-31.

6. Lee CC, Tsai MS, Fang CC, et al. Effects of pre-arrest comorbidities on 90-day survival of patients resuscitated from out-of-hospital cardiac arrest. Emerg Med J 2011:28:432-6.
7. Terman SW, Shields TA, Hume B, et al. The influence of age and chronic medical conditions on neurological outcomes in out of hospital cardiac arrest. Resuscitation 2015;89:169-76.

8. Beesems SG, Blom MT, van der Pas MH, et al. Comorbidity and favorable neurologic outcome after out-of-hospital cardiac arrest in patients of 70 years and older. Resuscitation 2015:94:33-9.

9. Ladha KS, Zhao K, Quraishi SA, et al. The deyo-charlson and elixhauser-van walraven comorbidity indices as predictors of mortality in critically ill patients. BMJ Open 2015;5:e008990.

10. Søholm H, Kjaergaard J, Bro-Jeppesen J, et al. Prognostic implications of level-of-care at tertiary heart centers compared with other hospitals after resuscitation from out-ofhospital cardiac arrest. Circ Cardiovasc Qual Outcomes 2015;8:268-76.

11. Maupain C, Bougouin W, Lamhaut L, et al. The CAHP (Cardiac Arrest Hospital Prognosis) score: a tool for risk stratification after out-of-hospital cardiac arrest. Eur Heart J 2016:37:3222-8.

12. Haukoos IS, Lewis RJ, Niemann JT. Prediction rules for estimating neurologic outcome following out-of-hospital cardiac arrest. Resuscitation 2004:63:145-55.

13. Ebell MH. Prearrest predictors of survival following in-hospital cardiopulmonary resuscitation: a meta-analysis. J Fam Pract 1992;34:551-9.

14. Ong ME, Shin SD, Tanaka H, et al. Pan-Asian Resuscitation Outcomes Study (PAROS): rationale, methodology, and implementation. Acad Emerg Med 2011;18:890-7.

15. Charlson ME, Pompei $\mathrm{P}$, Ales KL, et al. A new method of classifying prognostic comorbidity in longitudinal studies: development and validation. $J$ Chronic Dis 1987:40:373-83.

16. de Groot V, Beckerman H, Lankhorst GJ, et al. How to measure comorbidity. a critical review of available methods. J Clin Epidemiol 2003;56:221-9.

17. Extermann M. Measuring comorbidity in older cancer patients. Eur $\int$ Cancer 2000:199036:453-71.

18. Steyerberg E. Clinical prediction models: a practical approach to development, validation, and updating: Springer Science \& Business Media, 2008.

19. Scirica BM. Therapeutic hypothermia after cardiac arrest. Circulation 2013;127:244-50

20. Merchant RM, Soar J, Skrifvars MB, et al. Therapeutic hypothermia utilization among physicians after resuscitation from cardiac arrest. Crit Care Med 2006;34:1935-40.

21. Laver SR, Padkin A, Atalla A, et al. Therapeutic hypothermia after cardiac arrest: a survey of practice in intensive care units in the United Kingdom. Anaesthesia 2006:61:873-7.

22. Adrie C, Cariou A, Mourvillier B, et al. Predicting survival with good neurological recovery at hospital admission after successful resuscitation of out-of-hospital cardiac arrest: the OHCA score. Eur Heart J 2006;27:2840-5.

23. Ebell MH, Jang W, Shen Y, et al. Development and validation of the Good Outcome Following Attempted Resuscitation (GO-FAR) score to predict neurologically intact survival after in-hospital cardiopulmonary resuscitation. JAMA Intern Med 2013:173:1872-8 